\title{
ePraxis Nr. 1: ADSL-Anschluss in der Arztpraxis
}

\author{
Bei ADSL-Anschluss müssen die Patientendaten mit einer Firewall geschützt werden
}

Martin D. Denz, Heinz Bhend, Georg von Below

Sowohl im privaten als auch im beruflichen Alltag steigen die Ansprüche an die Leistungsfähigkeit im Telekommunikationsbereich. Das Bedürfnis nach schnellen Internet- und E-Mail-Verbindungen wächst in unserer Bevölkerung auch bei Ärztinnen und Ärzten. Dies ist einerseits auf dem Hintergrund der zunehmenden Integration von Informations- und Kommunikationsmitteln im privaten Alltag zu verstehen, anderseits ein Ausdruck der zunehmenden Digitalisierung im ärztlichen Alltag. In der Arztpraxis findet derzeit eine Aufrüstung mit Informatikmitteln statt, welche vorerst administrativ und ökonomisch motiviert ist (Büromatik, Terminplanung, Deckungsfragen, Rechnungsstellung). Künftig müssen die vorhandenen technischen Mittel konsequenter für ein medizinisch ausgerichtetes Informationsmanagement ausgeschöpft werden: schon stützen sich integrierte medizinische Versorgungsnetzwerke auf eine ortsunabhängig vernetzte elektronische Infrastruktur $a b$, das elektronische Patientendossier findet zunehmend Eingang in Klinik und Praxis. Bereits melden sich Patienten per E-Mail oder sie fragen nach einer Praxishomepage; der Zugriff auf medizinische Literaturdatenbanken wird zur Routine, das Nachschlagen im «World Wide Web» ersetzt das (veraltete) Lehrbuch. In naher Zukunft gehört deshalb ein ADSL-Anschluss zum allgemeinen Standard für den Internetzugang, auch in der Arztpraxis.

\section{Was ist ADSL?}

Mit ADSL kann man rascher im Internet surfen oder Daten austauschen. ADSL ist ein Breitbandanschluss zum Internet mit permanenter Verbindung. ADSL steht für «Asymmetric Digital Subscriber Line». Asymmetrisch bedeutet: Surfer können Daten viel schneller aus dem Internet herunterladen (Download), als ins Web hochladen (Upload). Je mehr Kilobit pro Sekunde, um so grösser das Tempo. Während man mit ISDN («Integrated Services Digital Network») eine Bandbreite von bis zu 64 Kilobit pro Sekunde nutzen kann, werden für ADSL Kilobitwerte (Download) von 256 bis zu 512 und mehr angegeben.

Diese Geschwindigkeitsangaben der Anbieter sind nur Bruttowerte, die tatsächlichen Nettowerte liegen um einiges tiefer. Aufgrund steigenden Drucks von Konsumentenseite wird dies bald ändern. Für den täglichen E-Mail-Verkehr und beim Surfen im Internet (z. B. Zugriff auf Medizinische Literaturdatenbanken) macht sich die eingeschränkte Maximalgeschwindigkeit kaum bemerkbar, höchstens beim Versenden oder Herunterladen grösserer Datenmengen während «Stosszeiten» im Datenverkehr.
Ein weiterer Vorteil eines ADSL-Anschlusses besteht darin, dass er über eine gewöhnliche Telefonleitung (analog oder ISDN) betrieben werden kann, dies 24 Stunden am Tag und ohne Unterbruch. Für diese Dienstleistung bezahlt man eine fixe monatliche Gebühr. Die Dauerverbindung bietet für den Praxisalltag praktische Vorteile, so zum Beispiel: die Telefonleitung bleibt frei, kein Einwählen ins Internet mehr nötig, keine Wartezeiten für den Verbindungsaufbau, automatische E-Mail-Briefkastenleerung, fixe Kostenkalkulation. Heute dank Breitbandanschluss bereits möglich, jedoch noch selten genutzt, sind attraktive und kostengünstige Möglichkeiten, wie normale Telefongespräche gebührenfrei über das Internet zu führen («Voice on IP») oder gar Videokonferenzen («Tele-Consulting»).

Als Alternative kann ein Kabelmodemanschluss eingesetzt werden (z.B. von Cablecom). Das Leistungsspektrum und der praktische Nutzen sind vergleichbar.

\section{Weshalb kann ADSL in der Praxis zu Problemen führen?}

Die permanente Verbindung ins Internet birgt jedoch auch Gefahren: über ADSL oder andere Breitbandanschlüsse besteht eine direkte Verbindung zwischen dem Internet und dem mit dem Internet in Kontakt stehenden PC. Lassen Benutzerinnen und Benutzer den Zugang zu ihrem PC (meist, ohne davon zu ahnen) zum Internet ungeschützt, droht die Gefahr, dass nicht nur dieser PC, sondern alle mit ihm über ein internes Netzwerk verbundenen Geräte, insbesondere Datenspeicher, von «unbefugten Eindringlingen» (z. B. Hacker) angegriffen, missbraucht oder zerstört werden. Ebenso können auf diesem Wege verbreitete Viren zu erheblichem Schaden führen. Der illegale Zugriff auf medizinische Daten, aber auch deren Veränderung oder Zerstörung ist nicht nur von Relevanz für den Datenschutz, ungünstigstenfalls kann er gar gesundheitliche Konsequenzen für die Patienten haben. Zudem kann sich ein derartiger Schadenfall auf eine betroffene Praxis - wie 
Tabelle 1

Auskunft über ADSL-Angebote mit Hardwarefirewall.

\begin{tabular}{llll} 
Anbieter & Hotline & E-Mail & Homepage \\
\hline Bluewin & 0844844884 & info@bluewin.ch & www.bluewin.ch \\
Sunrise & 0800111555 & business@sunrise.ch & www.sunrise.ch \\
\hline Green & 0564602323 & info@green.ch & www.green.ch \\
\hline HIN & 0848830740 & info@hin.ch & www.hin.ch/adsl \\
\hline
\end{tabular}

für jede Unternehmung - ökonomisch negativ auswirken (z. B. Betriebsgeheimnis, Produktivitätsausfall). Durch geeignete Vorkehrungen lassen sich ADSL- und andere Breitbandanschlüsse jedoch einfach und effektiv schützen (s.u.).

\section{Gesetzliche Hintergründe}

Patientendaten unterliegen einem besonderen Vertrauensverhältnis, das geschützt werden muss (Arztgeheimnis). Wer Personendaten bearbeitet oder ein Datenkommunikationsnetz zur Verfügung stellt, muss «angemessene technische und organisatorische Massnahmen» ergreifen, welche dem «gegenwärtigen Stand der Technik» entsprechen und «periodisch zu überprüfen» sind. Die Anforderungen an eine ärztliche Praxis unterscheiden sich prinzipiell nicht von anderen kleinen und mittleren Unternehmungen (KMU), welche ebenfalls die Verantwortung für sensible Daten tragen (z.B. Anwaltskanzlei). Auch für die Praxisorganisation braucht es ein Gesamtkonzept, das nicht nur den Datenschutz (Persönlichkeitsschutz), sondern auch die Datensicherheit (z. B. Backup gegen Datenverlust) berücksichtigen muss. Die Sicherheitsanforderungen an einen Breitbandanschluss (ADSL oder Kabelmodem) müssen im Verhältnis zu anderen Sicherheitsvorkehrungen stehen (Fax, Einbruchsicherung, Abschliessen der KG-Schränke usw). Gemäss aktuellem Stand der Technik gehört auch eine Hardwarefirewall dazu.

\section{Was kann ich tun?}

Der professionelle Umgang mit einem potentiellen Risiko trägt dazu bei, ein Problem sachlich zu lösen. Verschiedene Gefahren lauern im Internet - genauso wie in der Medizin. Ist dies ein Grund, auf die Nutzung des Internets und dessen Ressourcen zu verzichten? Ärztinnen und Ärzte können auf eigene professionelle Erfahrungen im Umgang mit Risiken zurückgreifen: beispielsweise sind aus dem Wissen über Infektionskrankheiten angemessene Verhaltensmass- nahmen ableitbar. Wir machen anlässlich einer Epidemie nicht unsere Praxis dicht, denn wir wissen, wie mit Infektionen sicher umzugehen ist; wir sind weiterhin in der Lage, unsere Patienten zu untersuchen und zu behandeln.

Im Zusammenhang mit der sinnvollen Nutzung der Informations- und Kommunikationstechnologien zugunsten der Medizin und ihrer Patienten geht diese Souveränität manchmal verloren: Kolleginnen und Kollegen verzichten mangels «Facharztausbildung für Internet» lieber auf die Internetnutzung ... Dabei ist es nicht notwendig, zum «Informatikprofi» zu mutieren, um einen ADSL-Anschluss zu nutzen! Das in diesem Artikel aufgeführte Basiswissen sowie die Kriterien samt Vergleichstabelle reichen aus, um sich zu entscheiden. Und genau wie sie sich im Berufsleben dafür entscheiden können, den Rat eines (z. B. infektiologischen) Konsiliararztes beizuziehen, steht es Ärztinnen und Ärzten frei, einen ADSL-Anbieter ihrer Wahl auszuwählen, der auf ihre spezifischen Bedürfnisse eingeht und ihre qualitativen Ansprüche erfüllt.

\section{Merkbox}

Auf folgende Punkte sollte jede Ärztin und jeder Arzt bei der Anschaffung von ADSL achten:

\section{Sicherheit}

Bei ADSL-Anschluss in der Arztpraxis müssen die Patientendaten mit einer Hardwarefirewall geschützt werden. Dies gilt auch für Kabelmodemanschluss und andere Breitbandanschlüsse mit permanenter Verbindungen zum Internet.

Hardwarefirewall

Wird eine Hardwarefirewall angeboten? Wird dieser Firewallschutz direkt durch den ADSL-Anbieter gewartet und überwacht? Was sind die einmaligen und die wiederkehrenden Kosten?

\section{Zusatzkosten}

Ist ein ADSL-Modem oder -Router im Angebot inbegriffen? Wie steht es mit den Aufschaltgebühren? Wird etwas für die Konfiguration und Wartung verlangt?

\section{Support}

Wird telefonischer Support angeboten? Werden dafür Minutentarife verrechnet? Wird eine direkte Telefonnummer angeboten? Sind die Mitarbeiter dort gut qualifiziert? 


\section{Keine Angst vor fachtechnischen Begriffen!}

\section{Firewall}

Eine Firewall ist ein Gerät (Hardware) oder eine Software, welche der Informatikinfrastruktur einer Arztpraxis wie ein Schutzwall («Brandmauer») vorgelagert wird, elektronische Angriffe erkennt und abblockt. Mit dem Einsatz einer Firewall ist ein professioneller Schutz vor Angriffen möglich. Firewalls auf Softwarebasis sind für eine Arztpraxis ungenügend und können allenfalls in Laptops zum Einsatz kommen.

\section{Router}

Ein Router ist ein Gerät, das den Datenfluss in einem Netzwerk regelt und die Verbindung zwischen zwei Netzwerken herstellt. Die Verbindung mehrerer PCs lokal oder über das Internet ist ein Netzwerk. Ein Router ist ein Kästchen, an dem die Datenkabel angeschlossen werden. Die darin enthaltene Elektronik kann auch in ein gemeinsames Gehäuse zusammen mit der Hardwarefirewall eingebaut werden, was den Erwerb eines separaten Routers erübrigt.

\section{ADSL-Router}

Es gibt zwar ADSL-Modems, welche direkt an einen PC angeschlossen werden können. Um einen ADSL-Anschluss richtig zu nutzen, braucht es jedoch einen Router. Weil die Funktionen von Router, Firewall und ADSL-Modem zunehmend in einem Gehäuse integriert werden, setzt sich immer mehr die zusammenfassende Bezeichnung «ADSL-Router» durch.

\section{Kabelmodem}

Im Gegensatz zu ADSL, das stets über eine Telefonleitung läuft, nutzt ein Kabelmodem den vorhandenen Fernsehanschluss. Die zu ergreifenden technischen (Router) und Sicherheitsmassnahmen (Hardwarefirewall) sind dieselben wie für ADSL.

Die Hardwarefirewalls bzw. ADSL-Router der neuesten Generation sind standardmässig derart konfiguriert, dass Attacken von aussen keine Chance haben (alle «Ports» geschlossen). Geräte, welche diese Kriterien erfüllen und von jedermann installiert werden können, kosten heute um Fr. 700.-, das Preisgefüge ist jedoch im Fluss und wird weiter sinken.

Das Angebot an Hardwarefirewalls ist gross genug, die Technologie ausgereift und erschwinglich, die Anschaffung und der Betrieb sind zumutbar. Eine Hardwarefirewall gehört deshalb zu jeder ärztlichen Praxis, welche über einen ADSL-Zugang verfügt. Dies gilt ebenso für Kabelmodemanschluss und andere Breitbandlösungen.

\section{«Do it yourself» oder «rundum sorglos»- Angebot?}

Wichtig ist, sich vor der Umrüstung auf einen ADSL-Anschluss zuerst gründlich über die Vorteile und Risiken von ADSL zu informieren. Ob Arztpraxis oder Spital: Die technischen Anforderungen (Hardwarefirewall) an die Sicherheitsmassnahmen sind grundsätzlich dieselben, die organisatorischen Massnahmen unterscheiden sich lediglich bezüglich ihrer Grössenordnung (siehe NZZ am Sonntag vom 10. August 2003: «So gefährden Schweizer Spitäler das Arztgeheimnis»).

Die Installation einer Hardwarefirewall ist einfach, technisch Versierte können dies mit wenig Aufwand selber vornehmen. Aber nicht alle Kolleginnen und Kollegen sind sogenannte «power users». Wie auch sonst im (Berufs-)Leben gilt der Grundsatz, die Grenzen der eigenen Verantwortung und Kompetenz zu erkennen. Dementsprechend werden sich viele Kolleginnen und Kollegen dafür entscheiden, diese Aufgabe einem spezialisierten Dienstleister zu übergeben. Es kommt hinzu, dass auch eine «Hardware»Firewall durch Softwareupdates auf den neusten Sicherheitsstand gebracht werden muss! Deshalb ist es wünschenswert, wenn der ADSL-Anbieter gleichzeitig ein «Sicherheitsgesamtpaket» samt Hardwarefirewall und Wartung anbietet. Und es macht Sinn, wenn dieser Anbieter Erfahrungen mit den spezifischen Bedürfnissen einer Arztpraxis oder vergleichbarer KMUs hat, weil deren Anforderungen sich von denjenigen der Allgemeinkunden unterscheiden.

\section{Qualitative Entscheidungskriterien}

Die einmal installierte Hardwarefirewall alleine ist keine Garantie für einen anhaltenden Schutz der Arztpraxis. Die Paketangebote machen erst Sinn im Zusammenspiel mit einem guten Support. Oft sagt der Preis allein wenig über die Gesamtqualität der Dienstleistung aus. Verschiedene Gesichtspunkte müssen berücksichtigt werden, wie zum Beispiel:

- Ist das ADSL-Angebot mit einem Hardwarefirewallschutz kombiniert?

- Ist ein ADSL-Router im Preis inbegriffen (Router mit integrierter Firewall)? 




- Steht eine direkte Telefonnummer für den Notfallsupport zur Verfügung?

- Ist ein Support auch ausserhalb der Bürozeiten gewährleistet?

- Werden für den telefonischen Support Minutentarife verrechnet?

- Sind die Mitarbeiter dort gut qualifiziert? Spezialisiert auf die Ärzteschaft?

- Wer übernimmt die Einstellungen der Firewall? Bereits vorkonfiguriert, bei Bedarf selber durchführbar oder auf Wunsch Installation vor Ort (Preis?) möglich?

- Wer übernimmt die Softwareaktualisierungen der Hardwarefirewall?

- Ist eine regelmässige Überwachung meines ADSL-Anschlusses (Sicherheitsüberprüfungen) samt Wartung (Firewallupdates) möglich; vor Ort oder per Fernwartung («Remote»)? Im Abonnementspreis inbegriffen oder Zusatzkosten?

\section{Fazit}

ADSL bietet für den Praxisalltag viele Vorteile, welche sehr bald eine Selbstverständlichkeit sein werden. In einer Arztpraxis, wo ein Internetzugang benötigt wird, macht bereits heute der Einsatz eines Breitbandanschlusses wie z.B. ADSL aus praktischen und ökonomischen Gründen Sinn. Vor der Anschaffung eines ADSL-Anschlusses in der ärztlichen Praxis muss aber zur Kosten-Nutzen-Abwägung unbedingt auch eine Risikoabwägung hinzukommen. Dabei ist es unumgänglich, sich sowohl der potentiellen Gefahren als auch der (einfachen) Lösungsmöglichkeiten bewusst zu sein:

1. Es ist fahrlässig, einen ADSL-Anschluss ohne angemessene Schutzmassnahmen zu nutzen.

2. Bei ADSL-Anschluss müssen eine Arztpraxis und insbesondere die dort vorhandenen Patientendaten unbedingt mit einer Hardwarefirewall geschützt werden!

Die nebenstehende Vergleichstabelle gibt einen Überblick über die erhältlichen ADSL-Paketangebote der wichtigsten Anbieter mit hilfreichen Entscheidungskriterien: 